\title{
An Epidemiological study and analysis of prognostic factors of testicular carcinoma
}

\author{
Manu Gupta ${ }^{1}$, Ashutosh Singh ${ }^{1}$ \\ ${ }^{1}$ Assistant Professor, General Surgery, KD Medical College Hospital \& Research Center, Mathura.
}

\section{Abstract}

Background: Testicular carcinoma is an uncommon malignancy overall accounting for 1-2\% of all tumours in men, testicular cancer is the most common malignancy in young men. The aim of the study was to analysis of prognostic factor of testicular carcinoma and determines their epidemiologic features, in order to provide a guideline for clinical treatment and follow ups. Subjects and Methods: Patient clinical data, including age, tumor position, pathological type, clinical stage, AFP, HCG and LDH levels, as well as postoperative treatment methods were identified and classified. Subsequently, the patients' conditions during the follow-up period following surgery were recorded. The survival rate was also calculated and prognosis was analyzed. Age upon operation, laterality of tumor, pathologic diagnosis, and preoperative serum AFP levels were reviewed. In GCT cases, preoperative serum AFP levels were analyzed to predict malignancy in different age groups. $\mathrm{P}<0.05$ was considered to indicate a statistically significant difference. Results: A total of 195 cases with testicular carcinoma were enrolled in this study. In $62.6 \%$ cases tumors were located on the right side, and in (37.4\%) tumors were located on the left side. The relation between the testicular seminoma and pathological Types shows that out of the 195 cases, 121(62.05\%) cases were confirmed with typicaltesticular seminoma, 52(26.7\%) cases with testicular seminoma combined with embryonal carcinoma and 22(11.3\%) cases withtesticular seminoma combined with embryonal carcinoma and teratoma. Conclusion: We concluded that the pathological types should be confirmed to determine the postoperative adjunctive treatment method and improve the postoperative survival rate. Epidemiological features of TC show a persistent lack of the knowledge about the symptoms of TC in the general population, in spite of extended health education.

Keywords: Testicular carcinoma, embryonal carcinoma, pathological type, preoperative serum AFP.

Corresponding Author: Dr. Manu Gupta, Assistant Professor, General Surgery, KD Medical College Hospital \& Research Center, Mathura.

Received: March 2019

Accepted: April 2019

\section{Introduction}

Incidence rates for testicular cancer (TC) occur in men age 25 to 39 years. With modern treatment, 5-year survival rates exceed $90 \% .^{[1,2]}$ The role of histologic type of TC and extent of disease (EOD) inidentifying groups for riskadapted treatment decisionsis well established. ${ }^{[3]}$

Alpha-fetoprotein (AFP) is the most important tumor marker for testicular tumors. Serum AFP levels are elevated in malignant GCTs. However, few articles reviewed the correlation of the preoperative AFP levels and the possibility of malignancy, especially the boundary AFP value of malignant GCTs. Testicular tumors are rare with an incidence from $0.3-2 / 100,000$ in children and adolescents or young adults, ${ }^{[4-6]}$ or $0.98-1.29 / 100,000$ in $<14$ years population. ${ }^{\text {[7] }}$ Most prepubertal testicular tumors are germ cell tumors (GCTs) comprising various histologic subtypes. Prognostic factors of testicular seminoma include age and pathology of the primary tumor: small vessel invasion, tumor size, and invasion of rete testis. ${ }^{[8]}$ Testicular cancer is primarily treated with inguinal orchiectomy that means the surgical removal of the affected testis. Pathological examination reveals histology of a seminoma in about $60 \%$ of the cases, with an increasing trend. ${ }^{[9]}$ About $80 \%$ of the diagnosed seminomas are classified as clinical stage I. This stage is characterized by the absence of metastases on radiologic examination and by normal or normalized testis tumor markers after orchiectomy. Data from large case series show that despite normal radiologic and laboratory examinations on primary diagnosis, about $15-20 \%$ of stage I seminoma patients will develop a tumor relapse in their further live. ${ }^{[10]}$ Therefore, early additional (adjuvant) therapies, namely adjuvant chemotherapy and adjuvant irradiation of the posterior abdominal wall, are used for stage I patients because they are able to lower the relapse risk to percentages $<5 \%$. ${ }^{[11,12]}$

The aim of the study was to analyse prognostic factors of testicular carcinoma and determines their epidemiologic features, in order to provide a guideline for clinical treatment and follow ups.

\section{Subjects and Methods}

All testicular carcinoma cases aged 20-60 who were diagnosed, in our institution located in Mathura, Uttar Pradesh. A total of 195 patients diagnosed with testicular carcinoma. The study was approved by the ethics 
committees at the K.D Medical College Hospital, Mathura. Patient clinical data, including age, tumor position, pathological type, clinical stage, AFP, HCG and LDH levels, as well as postoperative treatment methods were identified and classified. Subsequently, the patients' conditions during the follow-up period following surgery were recorded. The survival rate was also calculated and prognosis was analyzed. Age upon operation, laterality of tumor, pathologic diagnosis, and preoperative serum AFP levels were reviewed. In GCT cases, preoperative serum AFP levels were analyzed to predict malignancy in different age groups. Preoperative serum AFP levels were correlated with final histological outcome to determine the diagnostic accuracy of AFP for the preoperative detection of testicular malignancies. AFP values were taken common logarithm as lgAFP in graphing because of their wide distribution. Follow ups were mainly focused on patient postoperative treatment, survival state, and survival time. Survival time was calculated by months from the first time they underwent therapy until the last follow-up. Death events were defined as the end point. Patient survival or lost follow-up data were processed as censored data.

\section{Statistical analysis}

Statistical analysis was performed using SPSS 20.0 (SPSS, Inc., Chicago, IL, USA) statistical software. $\mathrm{P}<0.05$ was considered to indicate a statistically significant difference.

\section{Results}

A total of 195 cases with testicular carcinoma were enrolled in this study. In $62.6 \%$ cases tumors were located on the right side, and in $(37.4 \%)$ tumors were located on the left side. The relation between the testicular seminoma and pathological Types shows that out of the 195 cases, $121(62.05 \%)$ cases were confirmed with typical testicular seminoma, 52(26.7\%) cases with testicular seminoma combined with embryonal carcinoma and 22(11.3\%) cases with testicular seminoma combined with embryonal carcinoma and teratoma. The findings of typical testicular seminoma identified relatively higher. The relation between testicular seminoma and pathological types were shown in table 1, figure 1 .

Testicular seminoma is highly sensitive to radiotherapy and its recurrence rate following radiotherapy is extremely low. In the present study, 118 cases, accounting for $60.51 \%$ of cases, were treated with radiotherapy. The postoperative chemotherapy regimen administered was 3-cycle PEB regimen or 4-cycle EP regimen, and for patients in stage IIIC the 4-cycle PEB regimen was administered. The 3-year survival rate for postoperative radiotherapy, postoperative chemotherapy and postoperative radiotherapy combined with chemotherapy was analyzed in the present study. The results showed that all patients (100\%) survived for 3 years following treatment with postoperative radiotherapy. For patients who underwent postoperative chemotherapy an 83.3\% 3-year survival rate was observed. Table 2, fig. 2 shows the relation between the age and the occurrence of testicular carcinoma and table 3 shows the association between testicular carcinoma and treatment methods. The influence of clinical stage and postoperative treatment methods was statistically significant on patient prognosis $(\mathrm{P}<0.05)$.

Table 1: Association between testicular carcinoma and pathological types.

\begin{tabular}{|c|c|c|c|}
\hline Pathological type & Case & Proportion, $\%$ & $\begin{array}{l}\mathrm{P} \\
\text { value } \\
\end{array}$ \\
\hline $\begin{array}{l}\text { Typical testicular } \\
\text { carcinoma }\end{array}$ & 121 & 62.05 & \multirow{3}{*}{0.05} \\
\hline $\begin{array}{l}\text { Testicular seminoma } \\
\text { combined embryonal } \\
\text { carcinoma }\end{array}$ & 52 & 26.7 & \\
\hline $\begin{array}{l}\text { Testicular seminoma } \\
\text { combined embryonal } \\
\text { carcinoma as well as } \\
\text { teratoma }\end{array}$ & 22 & 11.3 & \\
\hline
\end{tabular}

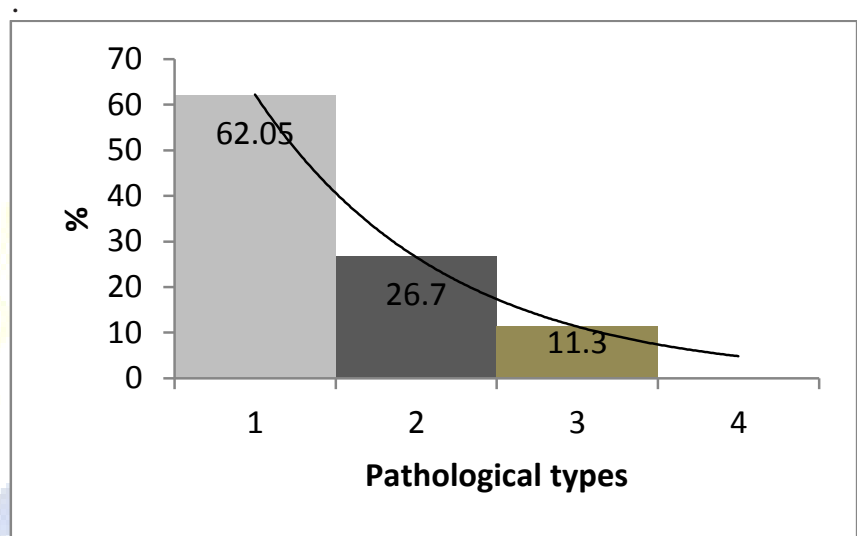

Figure 1: Association between testicular carcinoma and pathological types

Table 2: Relation between the age and the occurrence of testicular carcinoma.

\begin{tabular}{|c|c|c|c|}
\hline Age & Case & $\begin{array}{l}\text { Proportion, } \\
\%\end{array}$ & $P$ value \\
\hline $20-30$ & 39 & 20 & \multirow[t]{6}{*}{0.642} \\
\hline $31-40$ & 62 & 31.7 & \\
\hline $41-50$ & 53 & 27.17 & \\
\hline $51-60$ & 24 & 12.3 & \\
\hline$>60$ & 17 & 8.71 & \\
\hline Total & 195 & 100.0 & \\
\hline
\end{tabular}

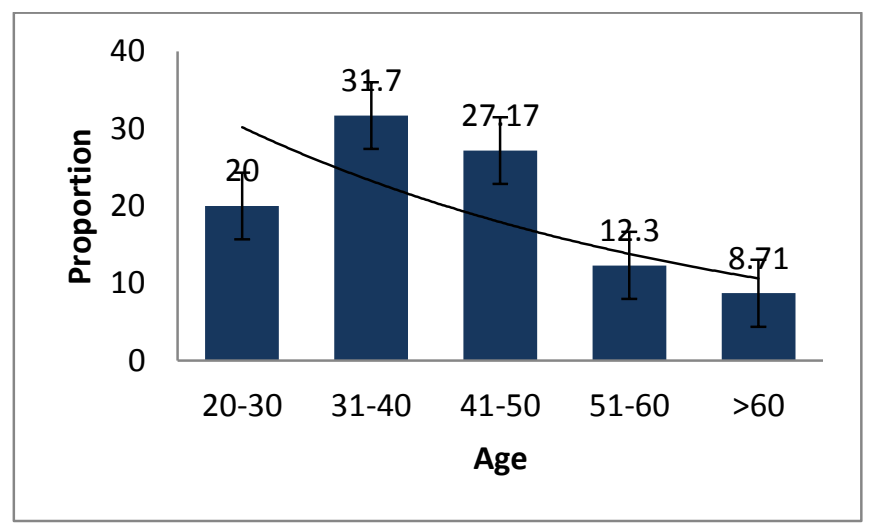

Figure 1: Relation between the age and the occurrence of testicular carcinoma 
Table 3: Relationship between testicularcarcinoma and treatment methods

\begin{tabular}{|c|c|c|c|}
\hline Treatment methods & Case & Proportion & $\begin{array}{l}\mathrm{P} \\
\text { value }\end{array}$ \\
\hline Postoperative radiotherapy & 118 & 60.51 & \multirow[t]{3}{*}{0.021} \\
\hline Postoperative chemotherapy & 56 & 28.71 & \\
\hline $\begin{array}{l}\text { Postoperative radiotherapy and } \\
\text { chemotherapy }\end{array}$ & 21 & 10.076 & \\
\hline
\end{tabular}

\section{Discussion}

Seminoma was the commonest tumour, constituting 34\%. It was observed between third and fourth decades. $8.82 \%$ cases were found in undescended testis. Melicow ${ }^{[12]}$ in his study of 55 cases of seminoma, found incidence of $5.5 \%$ in undescended testis. The symptoms were, painless scrotal swelling $(100 \%)$ and history of pain in $15.6 \%$ cases.

Embryonal carcinoma constituted $28 \%$ with peak age incidence during third and fourth decades. Patients presented with painless scrotal swelling in majority of cases. $7.14 \%$ cases were recorded in undescended testis. Kalra, et $\mathrm{al}^{[13]}$ made same observations. Microscopically tumour cells wore arranged in papillary form and there was moderate to marked degree of necrosis and haemorrhage. Mostofi14 observed same findings in his study. Current literature emphasizes that testicular germ cell tumors often occur on the right side. However, recent assessments suggested that there is no difference between the left and right sides. ${ }^{[15]}$ In present study $62.6 \%$ cases tumors were located on the right side, and in (37.4\%) tumors were located on the left side. The results showed that the incidence of right-side tumors was higher than that of the left-side tumors. Nonetheless, since there were a limited number of samples in the present study, whether right-side tumors had a higher frequency than those on the left side remains to be ascertained. A study with a larger sample group is likely to yield more accurate epidemiological and etiological results to determine and confirm whether the incidence of testicular seminoma is associated the position of the tumor. Currently, LDH, AFP, HCG are the most commonly used serum tumor markers for identifying testicular germ cell tumors. Radiotherapy has been the standard therapy for the treatment of seminoma in stages I, IIA and IIB. Postoperative adjuvant radiotherapy usually reduces the risk of local recurrence. ${ }^{[16]}$ However, in recent years, the importance of radiotherapy has been challenged. Zhang etal16 reported that systemic Chemotherapy was A safe and effective method for treating stage I seminoma patients following radical orchiectomy. However, other investigators have suggested radiotherapy increased the risk of second primary tumors, and did not recommend use of routine radiotherapy on patients in stage $\mathrm{I}^{[16]}$

Early diagnosis of TC is determined by the biologic features of the tumor, patients' knowledge about the disease, and the availability of GPs and specialists to analyze initial symptoms to determine a correct diagnosis and begin adequate treatment. ${ }^{[17]}$ Since the biologic features of tumors are hardly unbiased, other causes of treatment delay were analyzed in our study. The overall treatment delay (mean time of 150 days) shows that young males are generally poorly informed about the possibility of TC occurrence. They do not pay attention to the initial symptoms of the disease and most think that the enlargement of the testicle is a sign of their masculinity. Mortality decrease and survival improvement despite the incidence increase are the result of not only effective treatment, but also early diagnosis of each case. epidemiological features of TC show a persistent lack of the knowledge about the symptoms of TC in the general population, in spite of extended health education . However, the surprising result was the detection of an increased treatment delay caused by specialists (urologists, oncologists), who are responsible for most treatment approaches in this diagnosis. Therefore the only methods to decrease mortality and improve the survival of patients with TC may be early detection and risk-adapted treatment in specialized and adequately equipped centers experienced in TC management according to the appropriate guidelines and evidence-based medicine.

\section{Conclusion}

We conclude that the pathological types should be confirmed to determine the postoperative adjunctive treatment method and improve the postoperative survival rate.Epidemiological features of TC show a persistent lack of the knowledge about the symptoms of TC in the general population, in spite of extended health education.To decrease mortality and improve the survival of patients with TC may be early detection and risk-adapted treatment in specialized and adequately equipped centers experienced in TC management according to the appropriate guidelines and evidence-based medicine. The 3-, 5- and 10-year survival rate of patients with testicular seminoma following orchiectomy was closely associated with the clinical stage, pathological type and postoperative adjunctive therapy. For candidates of orchiectomy, the clinical stage of the tumor must be assessed first and surgery must be performed as early as possible.

\section{References}

1. Hayes-Lattin B, Nichols CR: Testicular cancer: prototypic tumor of young adults. SeminOncol 36:432-438, 2009

2. Verdecchia A, Francisci S, Brenner H, et al: Recent cancer survival in Europe: A 2000-02 periodanalysis of EUROCARE-4 data. Lancet Oncol 8:784796, 2007

3. Horwich A, Shipley J, Huddart R: Testicular germ-cell cancer. Lancet 367:754-765, 2006

4. Kay R. Prepubertal testicular tumor registry. J Urol 1993;150:671-4. Pauniaho SL, Salonen J, Helminen M, et al. Germ cell tumors in children and adoles-cents in Finland: trends over 1969-2008. Cancer Causes Control 2014;25:1337-41.

5. Lacerda HM, Akre O, Merletti F, et al. Time trends in the incidence of testicular cancer in childhood and young adulthood. Cancer Epidemiol Biomarkers Prev 2009;18:2042-5.

6. Alanee S, Shukla A. Paediatric testicular cancer: an updated review of incidence andconditional survival from the surveillance, epidemiology and end results database. BJU Int 2009;104:1280-3. 
7. Chung P, Daugaard G, Tyldesley S, Atenafu EG and Panzarella T: Evaluation of a prognostic model for risk of relapse in stage I seminoma surveillance. J Cancer Med 4: 155-160, 2015 (In Ch i nese).

8. Ruf CG, Isbarn H, Wagner W, Fisch M, Matthies C, Dieckmann KP. Changes in epidemiologic features of testicular germ cell cancer: age at diagnosis and relative frequency of seminoma are constantly and significantly increasing. UrolOncol. 2014;32(1):e1-6. doi:10.1016/j.urolonc.2012.12.002.

9. Mortensen MS, Lauritsen J, Gundgaard MG, Agerbaek M, Holm $\mathrm{NV}$, Christensen IJ, et al. A nationwide cohort study of stage I seminoma patients followed on a surveillance program. Eur Urol. 2014;66(6):1172-8. doi:10.1016/j.eururo.2014.07.001.

10. Fossa SD, Horwich A, Russell JM, Roberts JT, Cullen MH, Hodson NJ, et al. Optimal planning target volume for stage I testicularseminoma: a medical research council randomized trial Medical research council testicular tumor working group.J ClinOncol. 1999;17(4):1146.

11. Oliver RT, Mason MD, Mead GM, von der Maase H, Rustin GJ, Joffe JK, et al.Radiotherapy versus single-dose carboplatin in adjuvant treatment ofstage I seminoma: a randomised trial. Lancet. 2005;366(9482):293-300.doi:10.1016/s0140-6736(05)66984-x.

12. Melicow MM. Classification of tumours of testes. Clinical and pathological study based on 105 primary and 13 secondary cases in adults and 3 primary and 4 secondary cases in children. J Urol 1955; 73:547.

13. Kalra VB, Ramdeo IN, Kalra R. Testicular tumors clinicopathological study. Indian J Surg 1977; 39:226

14. Mostoti FK. Testicular Tumours epidemiologic, etiologic and pathologic features. Cancer 1973; 32:1186

15. Song $\mathrm{H}$ and Huang C: Clinical analysis on children testiculartumor (attached 55 case reports). Chin J Urol 25: 44-46, 2004.

16. Zhang X, Liu Z, Zhou F, Han H, Qin Z and Ye Y: A summaryof 10year experience in treating stage I testicular seminoma.Cancer 29: 98-101, 2010.

17. Hornak M, Ondrus D, Bardos A Jr (1987) Testicular tumors, the causes of therapeutic delay (in Slovak). PraktLe'k (Praha) 67:555556.

Copyright: (C) the author(s), 2019. It is an open-access article distributed under the terms of the Creative Commons Attribution License (CC BY 4.0), which permits authors to retain ownership of the copyright for their content, and allow anyone to download, reuse, reprint, modify, distribute and/or copy the content as long as the original authors and source are cited.

How to cite this article: Gupta M, Singh A. An Epidemiological study and analysis of prognostic factors of testicular carcinoma. Asian J. Med. Res. 2019;8(1):SG17-SG19.

DOI: dx.doi.org/10.21276/ajmr.2019.8.1.SG6

Source of Support: Nil, Conflict of Interest: None declared. 